Introduction/Background* Niraparib is a poly(ADP-ribose) polymerase (PARP) inhibitor approved as maintenance treatment for patients (pts) with newly diagnosed advanced or recurrent ovarian cancer following a response to platinum-based chemotherapy (CT) doublet. The PRIMA/ENGOT-OV26/GOG-3012 (NCT02655016) study showed that niraparib following firstline treatment improved progression-free survival (PFS) in the overall intention-to-treat (ITT) population (hazard ratio [HR] 0.62 ; 95\% CI 0.50-0.76).

Methodology This double-blind, placebo (PBO)-controlled, phase 3 trial evaluated niraparib in pts with newly diagnosed, advanced, high-grade serous or endometrioid ovarian, primary peritoneal, or fallopian tube cancer with a complete or partial response to first-line CT. Pts were considered to be at a high risk for disease progression based on their clinical characteristics. This post-hoc analysis presents the efficacy of niraparib, measured by PFS, based on time of surgery and residual disease status, and was not powered to determine differences among the subgroups.

Result(s)* Data cutoff was May 2019. In total, 733 pts were randomized in the PRIMA study. Efficacy outcomes by surgical timing, either primary debulking surgery (PDS) or interval debulking surgery (IDS), and postoperative residual disease status, either no visible residual disease (NVRD) or visible residual disease (VRD), are shown in table 1. Pts who underwent PDS or IDS had similar efficacy with niraparib maintenance treatment versus $\mathrm{PBO}$ in the ITT population (PFS HRs were 0.67 and 0.57 , respectively). Niraparib treatment reduced risk of progression by $42 \%$ in pts who received PDS and had VRD, 35\% in those with IDS and NVRD, and 59\% in those with IDS and VRD. Efficacy was not evaluable for pts with PDS and NVRD due to low sample size.

Conclusion* In this post-hoc analysis, the impact of residual disease after PDS or IDS on the efficacy of niraparib was comparable across subgroups. Pts with IDS and VRD had the highest reduction in the risk of progression.

\section{0 REAL-WORLD TREATMENT PATTERNS AND OUTCOMES WITH FIRST-LINE MAINTENANCE OLAPARIB FOR BRCAM ADVANCED OVARIAN CANCER: A PAN-EUROPEAN STUDY (OVAL-1)}

${ }^{1} \mathrm{C}$ Gourley*, ${ }^{2} \mathrm{~B}$ Asselain, ${ }^{3} \mathrm{D}$ Garbay, ${ }^{4} \mathrm{D}$ Lorusso, ${ }^{5} \mathrm{R}$ Miller, ${ }^{6} \mathrm{O}$ Condon, ${ }^{7} \mathrm{M}$ Kertous, ${ }^{8} \mathrm{C}$ Padrone, ${ }^{6} \mathrm{U}$ Rigney, ${ }^{6} \mathrm{~S}$ Dube. ${ }^{1}$ Cancer Research UK Edinburgh Centre, University of Edinburgh, Edinburgh, UK; ${ }^{2}$ Arcagy-GINECO, Paris, France; ${ }^{3}$ Clinique Tivoli Ducos, Bordeaux, France; ${ }^{4}$ Fondazione Policlinico Universitario Agostino Gemelli IRCCS, Rome, Italy; ${ }^{5}$ University College London Hospital, London, UK; ${ }^{6}$ AstraZeneca, Luton, UK; ${ }^{7}$ AstraZeneca, Courbevoie, France; ${ }^{8}$ AstraZeneca, Italy

\subsection{6/ijgc-2021-ESGO.350}

Introduction/Background* Standard therapy for advanced ovarian cancer (OC) includes radical debulking surgery followed by first-line platinum-based chemotherapy, although neo-adjuvant chemotherapy can be used. Most women with newly diagnosed advanced OC relapse within 3 years of standard treatment.

In SOLO1, patients with advanced OC and a BRCA1 and/ or BRCA2 mutation (BRCAm), in complete or partial response following first-line platinum-based chemotherapy, received maintenance olaparib or placebo for up to 2 years or until progression. At 5 -year follow-up, median progression-free survival (PFS) was 56 months with olaparib vs 14 months with placebo (hazard ratio 0.33 ; 95\% confidence interval $0.25-$ $0.43)$. The OVAL-1 study will provide evidence on real-world effectiveness of olaparib in patients with BRCAm advanced OC treated in the first-line maintenance setting in France, Italy and the UK.

Methodology Retrospective cohort, pan-European multicentre observational study with data abstracted from medical records at several time points until $\geq 3$ years after first olaparib dose (index date).

Eligible patients are adult females with a BRCAm and advanced (FIGO stage III/IV) OC, who received their first olaparib (tablet) dose between January 2019 and June 2020 in

Abstract 177 Table 1 Efficacy results by time of surgery and visible residual disease status

\begin{tabular}{|c|c|c|c|c|}
\hline & & ITT & NVRD (R0) & (R1/R2) \\
\hline All patients & $\begin{array}{l}\mathrm{N} \\
\mathrm{HR}(95 \% \mathrm{Cl}) \\
\mathrm{P} \\
\text { mPFS (nir vs PBO) } \\
\triangle \mathrm{mPFS} \\
\end{array}$ & $\begin{array}{l}733^{*} \\
0.62(0.5-0.76) \\
P<0.0001 \\
13.8 \text { vs } 8.2 \\
5.6\end{array}$ & & \\
\hline PDS & $\begin{array}{l}\mathrm{N} \\
\mathrm{HR}(95 \% \mathrm{Cl}) \\
\mathrm{mPFS} \text { (nir vs PBO) } \\
\Delta \text { mPFS } \\
\end{array}$ & $\begin{array}{l}236^{* *} \\
0.67(0.468-0.964) \\
13.7 \text { vs } 8.2 \\
5.5 \\
\end{array}$ & $\begin{array}{l}37 \\
\text { NE } \\
\text { NE } \\
\text { NE } \\
\end{array}$ & \begin{tabular}{|l|}
183 \\
$0.58(0.391-0.864)$ \\
11.8 vs 7.8 \\
4 \\
\end{tabular} \\
\hline IDS/NACT & $\begin{array}{l}\mathrm{N} \\
\mathrm{HR}(95 \% \mathrm{Cl}) \\
\mathrm{mPFS} \text { (nir vs PBO) } \\
\Delta \text { mPFS } \\
\end{array}$ & $\begin{array}{l}481^{* * *} \\
0.57(0.441-0.731) \\
14.2 \text { vs } 8.2 \\
6 \\
\end{array}$ & $\begin{array}{l}304 \\
0.65(0.461-0.91) \\
18.2 \text { vs } 10.9 \\
7.3 \\
\end{array}$ & \begin{tabular}{|l|}
149 \\
$0.41(0.269-0.620)$ \\
11.1 vs 5.6 \\
5.5 \\
\end{tabular} \\
\hline \multicolumn{5}{|c|}{$\begin{array}{l}\text { *16 patients had no debulking surgery. } \\
\text { **16 patients had unknown residual disease status. } \\
\text { ***28 patients had unknown residual disease status. } \\
\text { HR, hazard ratio; IDS, interval debulking surgery; ITT, intention-to-treat; mPFS, median progression- } \\
\text { free survival; NACT, neoadjuvant chemotherapy; NE, not evaluable; nir, niraparib; NVRD, no visible } \\
\text { residual disease; PBO, placebo; PDS, primary debulking surgery; VRD, visible residual disease. }\end{array}$} \\
\hline
\end{tabular}


the first-line maintenance setting via Early Access Programmes (Italy, UK), Temporary Use Authorization (France) or reimbursement following regulatory approval (Italy, UK). Main study endpoint is real-world PFS. Secondary endpoints include overall survival and response rates. The study will also describe surrogate measures of response and tolerability, including time to discontinuation, dose modifications (with reasons) and time to first and second subsequent treatment. Outcomes will be described by key subgroup status pre-index, including performance status, FIGO stage, BRCAm status, debulking surgery outcome and clinical response to chemotherapy. The study aims to include 350 patients. Retrospective data collection began in December 2020 and is planned to end by Q3 2023. As of April 2021, 69 patients have participated.

\section{UPLIFT (ENGOT-OV67/GOG-3048) A PIVOTAL COHORT OF UPIFITAMAB RILSODOTIN, A NAPI2B-DIRECTED ADC IN PLATINUM-RESISTANT OVARIAN CANCER}

${ }^{1} \mathrm{~N}$ Concin*, ${ }^{2,3}{ }^{\mathrm{E}}$ Hamilton, ${ }^{4} \mathrm{LM}$ Randall, ${ }^{5} \mathrm{~S}$ Banerjee, ${ }^{6} \mathrm{~L}$ Mileshkin, ${ }^{7,8} \mathrm{RL}$ Coleman, 9,10,11 B Monk, ${ }^{12,13,14,15,16,17}$ MR Mirza, ${ }^{18} \mathrm{~A}$ Savarese, ${ }^{19}$ I Ray-Coquard, ${ }^{20} \mathrm{JA}$ Perez Fidalgo, ${ }^{21} \mathrm{P}$ Bernardo, ${ }^{21} \mathrm{E}$ Putiri, ${ }^{21} \mathrm{R}$ Burger, ${ }^{21} \mathrm{R}$ Mosher, ${ }^{22} \mathrm{D}$ Richardson, ${ }^{23} \mathrm{D}$ Cibula, ${ }^{24} \mathrm{R}$ Madry, ${ }^{25} \mathrm{R}$ Chekerov, ${ }^{26} \mathrm{~T}$ Van Gorp. 'Medical University of Innsbruck, Austria, Department of Obstetrics and Gynaecology, Innsbruck, Austria; ${ }^{2}$ Sarah Cannon Research Institute, Nashville, United States; ${ }^{3}$ Tennessee Oncology, Nashville, United States; ${ }^{4}$ VCU Health System, MCV Physicians, Department of Obstetrics and Gynecology Oncology, Richmond, United States; ${ }^{5}$ The Royal Marsden Nhs Trust, Institute of Cancer Research, London, United Kingdom; ${ }^{6}$ Peter MacCallum Cancer Centre, Melbourne, Australia; ${ }^{7}$ Texas Oncology-The Woodlands, The Woodlands, United States; ${ }^{8}$ GOG Foundation; ${ }^{9}$ Arizona Oncology, US Oncology Network, Phoenix, United States; ${ }^{10}$ US Oncology, Gynecologic Program; ${ }^{11}$ GOG Partners; ${ }^{12}$ Rigshospitalet, København, Denmark; ${ }^{13}$ Nordic Society of Gynecological Oncology, Clinical Trial Unit, Denmark; ${ }^{14}$ European Network of Gynecological Trial groups; ${ }^{15}$ Gynecologic Cancer Inter-Group; ${ }^{16}$ European Society of Medical Oncology; ${ }^{17}$ European Society of Gynecological Oncology; ${ }^{18}$ Regina Elena National Cancer Institute, Rome, Italy; ${ }^{19}$ Léon Bérard Center, Lyon, France; ${ }^{20}$ Hospital Clínic Universitari, València, Spain; ${ }^{21}$ Mersana Therapeutics Inc, Cambridge, United States; ${ }^{22} \mathrm{OU}$ Health Stephenson Cancer Center, Oklahoma City, United States; ${ }^{23}$ Charles University, Czech Republic; ${ }^{24}$ Poznan University of Medical Sciences, Poznań, Poland; ${ }^{25}$ Charité - Universitätsmedizin Berlin, Berlin, Germany; ${ }^{26} \mathrm{Uz}$ Leuven, oncology, Leuven, Belgium

\subsection{6/ijgc-2021-ESGO.351}

Introduction/Background* Upifitamab rilsodotin (XMT-1536; UpRi), is a first-in-class Dolaflexin antibody-drug conjugate targeting NaPi2b, a sodium-dependent phosphate transport protein broadly expressed in solid tumors including high-grade serous epithelial ovarian cancer (OC). UpRi's safety and efficacy are being evaluated in a Phase I study (NCT03319628). Preliminary antitumor activity from an expansion cohort of heavily-pretreated OC patients has been reported (Hamilton et al, ESMO 2020). A data-cut of December 2020 demonstrated an ORR of $39 \%$ including 2 CRs and DCR of $81 \%$ in 26 OC patients with high NaPi2b expression (TPS $\geq 75$ ). The 2 patients achieving CR had previously been treated with bevacizumab and PARPi (Richardson et al, ASCO 2021, TPS5607). The prevalence of a TPS $\geq 75$ is greater than $60 \%$.

PROC remains a serious unmet medical need as available treatment options provide modest benefit of no more than $12 \%$ ORR and median OS less than 12 months. Based on encouraging anti-tumor activity of UpRi, UPLIFT was designed as a Phase 2 single-arm registration strategy for PROC as part of the ongoing study.

Methodology The UPLIFT cohort is enrolling patients with platinum resistant high grade serous ovarian, fallopian tube and primary peritoneal cancer with up to 4 prior lines of therapy. Prior bevacizumab is required for patients with 1 or 2 prior lines of therapy but is not required for patients with 3-4 prior lines of therapy. UPLIFT will enroll approximately 180 patients globally for 100 patients with high $\mathrm{NaPi} 2 \mathrm{~b}$ expression. UpRi is dosed intravenously at $43 \mathrm{mg} / \mathrm{m} 2$ every 4 weeks. Patients may enroll regardless of $\mathrm{NaPi} 2 \mathrm{~b}$ expression and regardless of baseline peripheral neuropathy. Baseline tumor samples (fresh or archived) will be collected for retrospective tumor tissue evaluation of $\mathrm{NaPi} 2 \mathrm{~b}$ expression.

Result(s)* The primary objective is assessment of objective response rate in patients with high $\mathrm{NaPi} 2 \mathrm{~b}$ expression. The cut-off for high $\mathrm{NaPi} 2 \mathrm{~b}$ expression is TPS $\geq 75$ and was based on data from the expansion cohort. Secondary endpoints include objective response rate in the overall population, duration of response, and adverse events.

Conclusion* This study is being conducted in collaboration with ENGOT and GOG. Patients will be enrolled globally. (NCT03319628).

\section{SURGICAL OUTCOME AS PROGNOSTIC FACTOR IN DIFFERENT HISTOLOGIC SUBTYPES OF OVARIAN CARCINOMA- ANALYSIS OF 7 PHASE III TRIALS BY AGO STUDYGROUP + ENGOT}

${ }^{1,2} \mathrm{~F} \mathrm{Heitz}^{*},{ }^{1,2} \mathrm{~A}$ Du Bois, ${ }^{2,3} \mathrm{~A}$ Reuß, ${ }^{4} \mathrm{E}$ Pujade-Lauraine, ${ }^{5} \mathrm{MR}$ Mirza, ${ }^{6} \mathrm{~S}$ Greggi, ${ }^{7} \mathrm{~N}$ Colombo, ${ }^{8} \mathrm{C}$ Marth, ${ }^{9} \mathrm{~B}$ Vergote, ${ }^{1,2} \mathrm{P}$ Harter, ${ }^{10}$ Ray-Coquard, ${ }^{11} \mathrm{~GB}$ Kristensen, ${ }^{2,12} \mathrm{~S}$ Mahner, ${ }^{13} \mathrm{AC}$ Hardy, ${ }^{14} \mathrm{~J}$ Sehouli, ${ }^{2,15} \mathrm{p}$ Wimberger, ${ }^{2,16} \mathrm{M}$ Eichbaum, ${ }^{2} \mathrm{G}$ Elser ${ }^{2,17} \mathrm{~W}$ Meier, ${ }^{2,18} \mathrm{~J}$ Pfisterer. ${ }^{1}$ Kliniken Essen-Mitte, Gynecology and Gynecologic Oncology; ${ }^{2}$ AGO Study Group, Wiesbaden; ${ }^{3}$ Philipps University Marburg, Coordinating Center for Clinical Trials of the Philipps-University of Marburg, Marburg, Germany; ${ }^{4}$ GINECO and Hôpital Dieu, Paris, France; ${ }^{5}$ NSGO \& Rigshospitalet, Dept. of Oncology; Copenhagen University Hospital," Copenhagen; ${ }^{6}$ MITO \& Istituto Nazionale per lo Studio e la Cura dei Tumori di Napoli, Gynecologic Oncology, Naples, Italy; ${ }^{7} \mathrm{MaNGO}$ \& European Institute of Oncology, Gynecologic Oncology, Milano, Italy; ${ }^{8}$ AGO-A \& Innsbruck Medical University, Department of Obstetrics and Gynecology, Innsbruck; ${ }^{9} B G O G$ \& University Hospitals Leuven, Dept. of Gynaecological Oncology, Leuven, Belgium; ${ }^{10}$ GINECO \& Centre Leon Bérard Université Claude Bernard Lyon Est, , Lyon, France; ${ }^{11}$ NSGO \& Oslo University Hospital, Oslo, Norway; ${ }^{12}$ University Hospital, LMU Munich, Department of Obstetrics and Gynecology; ${ }^{13}$ GINECO \& Hôpital Privé des Côtes d'Armor, Plérin; ${ }^{14}$ NOGGO \& Charité Universitätsmedizin Berlin, Campus Virchow, Dpt. Gynecology with Center for Oncological Surgery, Berlin, Germany; ${ }^{15}$ University Hospital Essen and University Hospital Carl Gustav Carus, TU Dresden, Department of Gynecology and Obstetrics, Dresden; ${ }^{16}$ Helios Dr. Horst Schmidt Kliniken Wiesbaden, Department of Gynecology, Wiesbaden; ${ }^{17}$ Düsseldorf University Hospital and Evangelisches Krankenhaus Düsseldorf, Department of Gynecology and Obstetrics, Düsseldorf, Germany; ${ }^{8}$ Gynecologic Oncology Center, Kiel, Germany

\subsection{6/ijgc-2021-ESGO.352}

Introduction/Background" Debulking surgery is the mainstay of treatment for patients (pts) with advanced epithelial ovarian cancer (EOC). Upfront surgery (PDS) with complete macroscopic resection (TR0) is associated with best survival while PDS to small residual disease (TR1-10) provides moderate benefit in high grade serous OC. The impact of resection status in other histological subtypes so far has not been defined and especially the role of TR1-10 is under debate. This analysis should help to better understand the interplay between histological subtype, surgical outcome, and prognosis.

Methodology Data of patients (>FIGO IIIB) from 7 AGOStudygroup led phase III multicentre trials (AGO-OVAR 3,5,7,9,11,12,15), (1995-2011) were pooled and analysed with focus on PDS resection status on overall survival (OS) in different histological subtypes: low grade (low grade serous or endometrioid), mucinous, clear cell, and high-grade (e.g. 